Fibrosis

\section{Postoperative fibrosis suppression}

\section{A L Schwartz}

\section{An alternative to intraoperative mitomycin C}

T he use of antimetabolites to modulate wound healing posttrabeculectomy has been a major advance in glaucoma filtering surgery. Initially, 5-fluorouracil and, more recently, mitomycin $\mathrm{C}$, have been used to dramatically improve success rates in patients at high risk for trabeculectomy failure, reducing the need for postoperative glaucoma medications. However, the use of antimetabolites has been accompanied by an increased risk of complications, including early and late bleb leaks, hypotony, maculopathy, and endophthalmitis. An alternative treatment that could be applied after surgery to rescue those blebs that appear at increased risk to fail would be valuable, especially if it would obviate the need for mitomycin C.

Fuller et al's study in this issue of the $B J O$ (p 1352) highlights a different approach to fibrosis suppression posttrabeculectomy. Their regimen involves the use of three agents taken orally: prednisone, a non-steroidal antiinflammatory agent, and colchicine. They used these drugs in a series of 77 of 551 eyes that had undergone trabeculectomy between 1978 and 1998. In none of these operations was an antimetabolite used. All patients had either primary open angle glaucoma or exfoliation syndrome and only two patients had a previous trabeculectomy.

These 77 eyes were selected for antifibrosis treatment because of an exaggerated postoperative healing response with increased bleb vascularity, a Tenon's cyst formation with elevated intraocular pressure, or a reduction in bleb size with visible fibrosis and threatened bleb failure. The antifibrosis regimen was started, on average, 11 days after surgery; the range was 7-30 days. The best response was in patients started within 2 weeks of surgery and six of the eyes required subsequent bleb needling. No cases of endophthalmitis, hypotony, maculopathy, late bleb leak, or serious systemic side effects from the postoperative regimen occurred.

Long term success results are very impressive as defined by their criteria Sixty nine of 77 eyes $(89.6 \%)$ were classified as successful with an IOP of less than or equal to $21 \mathrm{~mm} \mathrm{Hg}$. The Kaplan-Meier probability of success was 0.91 at 8 years. This compares quite favourably with the $52 \%$ success rate at 4 years, reported by Ehnrooth et al. ${ }^{1}$ However, today in a similar group of primary trabeculectomies, a pressure of $21 \mathrm{~mm}$ $\mathrm{Hg}$ post-trabeculectomy without consideration of disc and field status over time would not be classified a success by many ophthalmologists. The Advanced Glaucoma Intervention Study showed that patients required eye pressures less than $18 \mathrm{~mm} \mathrm{Hg}$ for all follow up visits to prevent progressive damage. ${ }^{2}$

Fuller et al recommend that the oral medications be taken three times a day to be maximally effective. Given their potential side effects, the use of three systemic agents in an older age group population is somewhat worrisome. Dosages were tailored to patients' size, age, general health, and degree of possible bleb failure and they were reported to be well tolerated, with only seven patients requiring an oral histamine-2 blocker (ranitidine) to counteract gastrointestinal upset. One wonders why this regimen has not been more widely embraced. $^{3}$

This series is noteworthy because of its unique therapeutic approach which may help to reduce the growing incidence of complications associated with the use of mitomycin C. This regimen of fibrosis suppression has particular appeal in primary trabeculectomies. Bindish et al reported 123 eyes that underwent primary trabeculectomy with varying concentrations and durations of mitomycin C application. ${ }^{4}$ Their complications included hypotony (IOP $<6 \mathrm{~mm} \mathrm{Hg}$ ) in $42.2 \%$ of eyes with a mean follow up of 26.1 months and hypotony maculopathy in $8.9 \%$ of eyes. It also could be used after mitomycin $\mathrm{C}$ trabeculectomy as adjunctive therapy to try to rescue a potentially failing filter if there were signs of early bleb failure.

For many eyes, this "exaggerated" healing phase may be part of an encapsulated bleb phase which responds well to conservative topical aqueous suppressant therapy. ${ }^{5}$ For others, it may represent a scarring process leading to bleb failure. We do not know how many of these blebs would fail and how many would function if this antifibrosis regimen was not initiated. A prospective randomised study comparing conventional postoperative topical therapy versus the antifibrosis regimen of postoperative prednisone, a non-steroidal antiinflammatory, and colchicine would clarify its true benefit.

Br J Ophthalmol 2002;86:1323

\section{Author's affiliations}

A L Schwartz, 5454 Wisconsin Avenue, Suite 950, Chevy Chase, MD 20815, USA

\section{REFERENCES}

1 Ehnrooth G, Lehto I, Puska P, et al. Long-term outcome of trabeculectomy in terms of intraocular pressure. Acta Ophthalomol Scand 2002;80:267-71.

2 The Advanced Glaucoma Intervention Study Investigators. Advanced Glaucoma Intervention Study: 7. The relationship between control of intraocular pressure and visual field deterioration. Am J Ophthalmol 2000; 130:429-40.

3 Molteno ACB, Straughan JL, Ancker E. Control of bleb fibrosis after glaucoma surgery by anti-inflammatory agents. S Afr Med J 1976;50:881-5.

4 Bindish R, Condon GP, Schlosser JD, et al. Efficacy and safety of mitomycin-C in primary trabeculectomy. Ophthalmology 2002; 109:1336-41.

5 Scott DR, Quigley HA. Medical management of a high bleb phase after trabeculectomies. Ophthalmology 1988;95: 11 169-73. 

choroidopathy? Are we assisted by isolated clinicopathological reports in our understanding of the pathogenesis of uveitis? Reverting back to the animal A D Dick

\section{Pathological correlate confirms clinical and experimental observations in posterior uveitis}

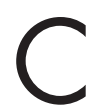
ontinuing our understanding of the basic pathobiology of noninfectious posterior segment intraocular inflammatory conditions has relied largely on experimental models of uveitis, such as experimental autoimmune uveoretininitis (EAU). Arguably such models are not well supported by human data in that there is still no definitive evidence of a role for retinal autoantigens in posterior uveitic conditions, ${ }^{12}$ despite us moving on to clinical trials of tolerance induction. ${ }^{3}$ On the other hand the models are useful. The pathological changes observed appear to explain what we may see clinically-namely, vitritis, retinal vasculitis, chorioretinal leucocytic infiltrates, and optic nerve head and macula oedema. ${ }^{45}$ As such we are able to discern common immune mediated processes that lead to inflammation, in particular $\mathrm{T}$ cell and macrophage behaviour, cytokine mediation of inflammatory response, and other immune regulatory mechanisms in play in the eye. ${ }^{6}$ To this end, preclinical studies of novel immune modulatory agents (a classic example being cyclosporin ${ }^{7}$ ) have now been successfully translated into clinical practice. ${ }^{8}$ There are caveats in our interpretations. Although in animal models the photoreceptors are the target tissue for retinal antigen specific autoreactive $\mathrm{T}$ cells precipitating the non-specific inflammatory process that follows, ${ }^{910}$ in humans there is little evidence that photoreceptors are primarily destroyed during posterior uveitis. Definitively, therefore, we require support of human pathological studies to confirm pathological processes we observe in experimental models. To date such an aim has been restricted because specimens have frequently been of end stage disease, which does not allow us to understand the evolution of ocular inflammation. Moreover, in humans non-infectious ocular inflammation represents a spectrum of conditions, the study of which may not permit an iteric approach to investigating pathology. We thus resort to modulating experimental models by varying antigen, antigen dose, species, and strain of animals used so as to mimic the spectrum of clinical non-infectious ocular inflammatory conditions, in particular Vogt-Koyanaga-Harada disease, sympathetic ophthalmia, and birdshot retinochoroidopathy. These conditions demonstrate a significant HLA association with both MHC class II molecules in the former two $^{11}$ and MHC class I molecules in birdshot retinochoroidopathy, ${ }^{12}{ }^{13}$ providing some relevance to the notion of an autoimmune pathogenesis. If we were to consider animal models as a true representation of posterior uveitis in humans and not just providing excellent experimental conditions to investigate general immunobiological processes and cell behaviour in the eye then we need more pathological information on specific uveitic conditions in order to translate our experimental findings more purposefully.

\section{We require support of human pathological studies to confirm pathological processes we observe in experimental models}

In this issue of the BJO (p 1439) Gaudio and co-workers have enlightened our understanding of an archetypical uveitis, birdshot retinochoroidopathy. Their clinicopathological description of "non-end stage" disease in an HLA A29 patient is timely. Birdshot retinochoroidopathy was initially described in patients with profuse retinal vascular leakage with resultant retinal, macular, and disc oedema ${ }^{14}{ }^{15}$ with a poor prognosis over many years. ${ }^{16}$ The recently described animal model of spontaneous retinopathy in HLA-A29 transgenic mice ${ }^{17}$ supports the high specificity and sensitivity of HLA-A29 positivity in the diagnosis of birdshot retinochoroidopathy, ${ }^{12}{ }^{13}$ and the increased relative risk of developing the disease when this allele is present. HLAA29 Tg mice are characterised histologically by changes that are almost identical to those described in the report by Gaudio et al, including perivasculitis, vitritis, leucocytic infiltration of the optic nerve head, and choroiditis. More immunohistochemical information of, for example, cell phenotype, microglial activation, GFAP reactivity, and cytokine profile would have assisted in comparing findings with those of experimental models. model EAU, the most potent autoantigens that induce disease are soluble $\mathrm{S}$-antigen ( $\mathrm{S}-\mathrm{Ag}$ ) and interphotoreceptor retinoid binding protein (IRBP). Consequently, $\mathrm{T}$ cell responses in humans to these antigens have been studied extensively in a variety of uveitic conditions, and have been found particularly prevalent in birdshot retinochoroidopathy. ${ }^{18}$ There is now conceivably more relevance of S-Ag reactivity in uveitis. Firstly, there is biochemical evidence that two peptides from the carboxy terminus of S-Ag bind efficiently with the peptide binding motif of HLA-A29. ${ }^{19}$ Secondly, the previous clinicopathological report of birdshot chorioretinopathy exhibited strong $\mathrm{T}$ cell proliferative responses to $\mathrm{S}-\mathrm{Ag}^{18}$ despite being no pathological features that truly paralleled the classic clinical features of birdshot during its evolution or paralleled the pathological observations in the HLA-A29 Tg mouse (the eye, however, was phthisical and frustratingly the patient was also HLA-A29 negative). Thirdly, although both reports show no conclusive evidence of photoreceptor involvement as seen during the development and end stage of inflammation in animal models, there was a significant granulomatous response in the outer retina in late stages of disease, which could be interpreted as targeting the photoreceptors. Clinically, end stage disease is highlighted by chorioretinal atrophy and reduced ERG responses but we still lack the definitive evidence to support the primary targeting of photoreceptors, as the clinical findings may be explained by vasculitis and ischaemia.

Finally, if we were to conclude that clinicopathological studies in uveitis or more pertinently birdshot retinochoroidopathy are represented well by animal models how, in the case of an MHC class I restricted disease, do we generate S-Ag specific CD4+ $\mathrm{T}$ cell activation and retinal inflammation? Historically the evidence for molecular mimicry, where infectious agents cross react with self determinants on, for example, HLA A29 is poor, despite isolated clinical reports. ${ }^{20}$ The combination, however, of this current report and the generation of HLA-A29 Tg mice that demonstrate pathological features which strikingly resemble this present case offer an exciting future to develop our understanding of the pathogenesis of birdshot retinochoroidopathy and the role of retinal antigens, the results of which may then be translated to other uveitic conditions.

Br J Ophthalmol 2002;86:1323-1325 


\section{Author's affiliations \\ A D Dick, Division of Ophthalmology, \\ University of Bristol, UK; a.dick@bristol.ac.uk \\ REFERENCES \\ 1 De Smet MD, Chan CC. Regulation of ocular inflammation - what experimental and human studies have taught us. Prog Retin Eye Res 2001;20:761-97. \\ 2 De Smet MD, Bitar G, Mainigi S, et al. Human S-antigen determinant recognition in uveitis. Invest Ophthalmol Vis $\mathrm{Sc}$ 2001;42:3233-8. \\ 3 Nussenblatt RB, Caspi RR, Mahdi R, et al. Inhibition of S-antigen induced experimental autoimmune uveoretinitis by oral induction of tolerance with S-antigen. J Immunol 1990;144: 1689-95. \\ 4 Forrester JV, Liversidge J, Dua HS, et al. Experimental autoimmune uveoretinitis: a model system for immunointervention: a review. Curr Eye Res 1992;11 (Suppl):33-40 \\ 5 Forrester JV. Duke-Elder lecture: New concepts on the role of autoimmunity in the pathogenesis of uveitis. Eye $1992 ; 6: 433-41$ \\ 6 Dick AD. Immune regulation of uveoretinal inflammation. Int Ophthalmol 1999;30: 187-202.}

7 Nussenblatt RB, Rodrigues MM, Salinas-Carmona MC, et al. Modulation of experimental autoimmune uveitis with cyclosporin A. Arch Ophthalmol 1982;100:1146-9.

8 Nussenblatt RB, Salinas-Carmona $M$ Waksman $\mathrm{BH}$, et al. Cyclosporin $\mathrm{A}$ : alterations of the cellular immune response in S-antigen- induced experimental autoimmune uveitis. Int Arch Allergy Appl Immunol 1983;70:289-94.

9 Caspi RR, Roberge RG, McAllister CG, et al. $T$ cell lines mediating experimental autoimmune uveoretinitis (EAU) in the rat. $J$ Immunol 1986:136:928-32.

10 Caspi RR, Chan CC, Fujino F, et al. Recruitment of antigen-nonspecific cells plays a pivotal role in the pathogenesis of a $T$ cell-mediated organ-specific autoimmune disease, experimental autoimmune uveoretinitis. J Neuroimmunol 1993;47:177-83

11 Kilmartin DJ, Wilson D, Liversidge J, et al. Immunogenetics and clinical phenotype of sympathetic ophthalmia in British and Irish patients. Br J Ophthalmol 2001:85:281-6.

12 Priem HA, Kijlstra A, Noens L, et al. HLA typing in birdshot chorioretinopathy. Am J Ophthalmol 1988;105:182-5.
13 Priem HA Oosterhuis JA. Birdshot chorioretinopathy: clinical characteristics and

14 Ryan SJ, Maumenee AE. Birdshot retinochoroidopathy. Am J Ophthalmol 1980:89:31-45.

15 Kaplan HJ, Aaberg TM. Birdshot retinochoroidopathy. Am J Ophthalmol 1980;90:773-82.

16 Oh KT, Christmas NJ, Folk JC. Birdshot retinochoroiditis: long term follow-up of a chronically progressive disease. Am J Ophthalmol 2002;133:622-9.

17 Szpak Y, Vieville JC, Tabary $T$, et al. Spontaneous retinopathy in HLA-A29 transgenic mice. Proc Natl Acad Sci USA transgenic mice. Proc
2001;98:2572-6.

18 Nussenblatt RB, Mittal KK, Ryan S, et al Birdshot retinochoroidopathy associated with HLA-A29 antigen and immune responsiveness to retinal S-antigen. Am J Ophthalmol 1982:94:147-58.

19 Boisgerault F, Khalil I, Tieng V, et al. Definition of the HLA-A29 peptide ligand motif allows prediction of potential T-cell epitopes from the retinal soluble antigen, a candidate autoantigen in birdshot retinopathy. Proc Nat Acad Sci USA 1996:93:3466-70.

20 Suttorp-Schulten MS, Luyendijk L, van Dam $A P$, et al. Birdshot chorioretinopathy and Lyme borreliosis. Am J Ophthalmol 1993;115:149-53.
Our new online system, "Bench> Press," was designed by HighWire Press, the same group that designed our website. Extensive research was conducted by HighWire before developing Bench>Press, but everyone involved realises it is a system in evolution. HighWire has been extremely responsive to our recommendations for changes. The vast majority of these have come about as the result of suggestions from our authors and reviewers. Minor additions and changes are likely to continue over the next several months. We welcome constructive criticism and suggestions that will help improve the system.

A new, more detailed set of instructions for using the system has been made available recently-please $\log$ on to our website-www.bjophthalmol.com, then click on "Submitting a manuscript" and go to "For details on how to submit your manuscript online, via Bench>Press." We hope these instructions will simplify the process for both authors and reviewers. Additionally, we are developing a problem solving page for users of $\mathrm{BJO}$ - http:// bjo.bmjjournals.com/misc/problemsolver. shtml. Although we now believe these more detailed instructions should solve most problems authors and reviewers have experienced in the past, we urge users of Bench>Press not to hesitate in contacting our Bristol office (anne.williams@bristol. ac.uk) or our San Francisco office (choyt@itsa.ucsf. edu) if problems arise that cannot be solved with the aid of these new instructions. "original article" was reduced to 21 days the average time required for review, revision, and acceptance of an origina article was only 27 days. For "letters to efficient. Secondly, it allows us to roucentury sending hard copies of manuStates. We hope this will not rema submit papers to the journal to $d$ authors in countries from whic manuscripts. The new online system journal a truly international journal of ophthalmology - not only with regard to its editorial board but also to its authors, reviewers, and, most importantly, readers. 
It will undoubtedly take a bit more time for all authors and reviewers to feel perfectly comfortable with our Bench> Press system. Ultimately, we believe all authors and reviewers will find it quicker and more efficient than the old system of hard copy manuscripts. In the meantime we apologise for any frustra- tion the system has caused any of its users. Rest assured even the editors have endured a few days (not many) of second thoughts. Yet, we will do everything possible to steer the transition smoothly forward until the online system is problem free. Moreover, we will attempt to minimise the drama involved in its use.
We firmly believe the end product will be worth the effort.

\section{Author's affiliations}

C S Hoyt, Editor, San Francisco, California, USA

\section{New BJO online submission and review system}

The Editors of British Journal of Opthalmology are pleased to inform authors and reviewers of its new online submission and review system. Bench $>$ Press is a fully integrated electronic system which uses the internet to allow rapid and efficient submission of manuscripts, as well as the entire peer review process to be conducted online.

Authors can submit their manuscript in any standard word processing software. Graphic formats acceptable are: .jpg, .tiff, .gif, and eps. (Nb. Multipage PowerPoint presentations are NOT acceptable.) Text and graphic files are automatically converted to PDF for ease of distribution and reviewing purposes. Authors are asked to approve their submission before it formally enters the reviewing process.

To access the system click on "SUBMIT YOUR MANUSCRIPT HERE" on the BJO homepage: http://www.bjopthalmol.com/, or you can access Bench>Press directly at http://submit-bjo.bmijournals.com/.

We are very excited with this new development, it really is simple to use and should be a big improvement on the current peer review process. Full instructions can be found on Bench>Press and BJO online. Please contact Natalie Davies, Project Manager, ndavies@bmigroup.com for further information.

\section{Pre-register}

We would be grateful if all British Journal of Opthalmology authors and reviewers pre-registered with the system. This will give you the opportunity to update your contact and expertise data, allowing us to provide you with a more efficient service.

\section{Instructions For Registering}

1. Enter http://submit-bjo.bmijournals.com

2. Click on "Create a New Account" in the upper left hand side of the Bench>Press

homepage.

3. Enter your email address in the space provided.

4. Choose a password for yourself and enter it in the spaces provided.

5. Complete the question of your choice to be used in the event you cannot remember your password at a later time.

6. Click on the "Save" button at the bottom of the screen.

7. Check the email account you registered under. An email will be sent to you with a verification number and URL.

8. Once you receive this verification number, click on the URL hyperlink and enter the verification number in the relevant field. This is for security reasons and to check that your account is not being used fraudulently.

9. Enter/amend your contact information, and update your expertise data.

10. Please note: You only need to create a new account once. If you submit to another

BM Publishing Group journal you can use the same email address and password 